\title{
Investigating the antibacterial potential of agarose nanoparticles synthesized by nanoprecipitation technology
}

\author{
Rukhsana Satar ${ }^{1}$, Syed Ahmed Iizhar ${ }^{2}$, Mahmood Rasool ${ }^{3}$, Peter Natesan Pushparaj ${ }^{3}$, Shakeel \\ Ahmed Ansari ${ }^{*}$ \\ ${ }^{1}$ Ibn Sina National College for Medical Sciences, Department of Biochemistry, Jeddah-21418, Kingdom of Saudi Arabia \\ ${ }^{2}$ Ibn Sina National College of Medical Studies, Department of Pharmaceutical Sciences, Jeddah-21418, Kingdom of \\ Saudi Arabia \\ ${ }^{3}$ King Abdulaziz University, Center of Excellence in Genomic and Medicine Research, Jeddah-21589, Kingdom of Saudi \\ Arabia \\ "Corresponding author: e-mail: shakeel.cegmr@gmail.com
}

\begin{abstract}
Herein, an effort was made to investigate the antibacterial potential of agarose nanoparticles (ANPs) and poly(quaternary ammonium) modified ANPs (mANPs) against Escherichia coli (gram-negative bacterium) and Staphylococcus aureus (gram positive bacterium) in liquid systems as well as on agar plates. ANPs were synthesized by nanoprecipitation technology and characterized by XRD, TEM, TGA, DTA and DLS. The particle size estimated was $30 \mathrm{~nm}$ while atomic force microscopy was used to observe the interaction of ligand on ANPs. Antimicrobial characterization was monitored by colony forming units (CFU) as a function of ANPs concentration on agar plates. It was observed that ANPs showed $15 \times 10^{9} / \mathrm{ml}$ CFU after 24 hours of incubation at $20 \mathrm{mM}$ ANPs concentration while the modified ANPs exhibited $21 \times 10^{9} / \mathrm{ml} \mathrm{CFU}$ under similar incubation conditions. Moreover, zone of inhibition (ZOI) was 2.9 and $3.8 \mathrm{~cm}$, respectively for $E$. coli by ANPs at 0.2 and $0.4 \mathrm{mM}$, respectively while it was 3.2 and $3.8 \mathrm{~cm}$ respectively by modified ANPs under similar conditions. Similarly, ZOI for S. aureus by ANPs at 0.2 and $0.4 \mathrm{mM}$ was observed at 3.1 and $4.0 \mathrm{~cm}$, respectively, while these values were 3.5 and $4.1 \mathrm{~cm}$, respectively for modified ANPs under similar incubation conditions.
\end{abstract}

Keywords: agarose nanoparticles, antibacterial properties, surface modification, nanotechnology.

\section{INTRODUCTION}

Owing to their excellent opto-electronics and several biomedical applications, last decade had attracted researchers to synthesize polymer based metal nanoparticles for diverse applications $\mathbf{s}^{\mathbf{1}, 2}$. In this regard, biopolymers have become the preferred choice as polymer matrices due to their easy availability, inexpensive and environmental friendly nature ${ }^{3,4}$. Moreover, the presence of oxygen rich functionalities (eg. cellulose, starch and alginic acid) and their affinity towards metals make them ideal candidates for stabilizing nanoparticles ${ }^{5}$.

Interactions between metals and microbes have emerged as an important aspect in several biotechnological applications including biominearlization, bioremediation, biotechnology and microbial corrosion, thereby leading to the development of nanoparticle based antimicrobial agents. They exhibited strong inhibitory and bactericidal effects as well as broad spectrum of antimicrobial activities $^{6,7}$. It should be noted that due to the development of resistant strains of bacteria to bactericides and antibiotics, massive need arises to explore novel methods to formulate new types of safe and cost effective material against such microbes ${ }^{8}$. Hence, the antimicrobial nature of nanoparticles showed promising feature which can be exploited in the medical field.

Hence, in this study, efforts were raised to develop a simple, efficient and inexpensive procedure to evaluate antibacterial effect induced by poly (quaternary ammonium) functionalized ANPs.

\section{RESEARCH METHODOLOGY}

Synthesis and modification of agarose nanoparticles

Agarose nanoparticles [ANPs] were prepared by nanoprecipitation technique. Briefly, agarose solution was prepared by dissolving it in dimethyl sulfoxide $(1 \% \mathrm{w} / \mathrm{v})$ followed by its addition to $1.5 \% \mathrm{w} / \mathrm{v}$ polyvinyl alcohol $(30 \mathrm{ml})$ by using magnetic stirrer (Tarson spinnot digital) at $1000 \mathrm{rpm}$ for $10 \mathrm{~min}$ at room temperature. Finally, n-butanol ( $2 \mathrm{ml})$ was added and stirred continuously for $5 \mathrm{~min}$. The obtained nanoparticles were washed thrice by distilled water in order to remove byproducts bound to nanoparticles. They were freeze-dried and compressed at room temperature for fewminutes and left at room temperature for two hours. In order to modify the surface of obtained ANPs, they were suspended in poly (quaternary ammonium) salt in a shaker at 250 $\mathrm{rpm}$ for $4 \mathrm{~h}$. The activated support was removed by centrifugation, washed twice with deionized water and kept at room temperature for further use.

\section{XRD, TEM, TGA and DTA of the synthesized ANPs}

These experiments have been discussed in detail in the manuscript entitled "Functionalization of agarose nanoparticles by galactose for efficient production of lactose-free dairy products" accepted in Brazilian Journal of Chemical Engineering having manuscript no. BJCE-2015-0319R1.

\section{DLS of ANPs and AFM of modified nanomatrix}

A laser diffraction method based on Mie-scattering theory was used to determine the particle size distribution of the powder. In order to find out the particles size distribution, ANPs were dispersed in water by horn 
type ultrasonic processor followed by the particles size distribution in computer controlled particle size analyzer [Malvern Instruments Nano ZS]. In another experiment, tapping mode AFM experiments of poly (quaternary ammonium) modified ANPs was performed using commercial etched silicon tips as AFM probes by exposing the modified nanoparticle with typical resonance frequency of ca. $300 \mathrm{~Hz}$ (RTESP, Veeco, Japan).

\section{Disc Diffusion Method}

Antibacterial assays were performed by standard disc diffusion method in which nutrient broth $(1 \mathrm{~g}$ beef extract, $1 \mathrm{~g}$ peptone, $0.5 \mathrm{~g} \mathrm{NaCl}$ dissolved in $100 \mathrm{ml}$ of double distilled water) was used to cultivate bacteria. The media was autoclaved and cooled, and poured in petri dishes and kept for 30 minutes for solidification. After 30 minutes, the fresh overnight cultures of inoculums $(100 \mu \mathrm{l})$ of two different cultures were spread on to solidified nutrient agar plates. Sterile paper discs $(5 \mathrm{~mm}$ diameter) made of Whatman filter paper (dipped in 50 $\mathrm{mg} /$ litre ANPs) was placed in each plate. The cultured agar plates were incubated at $37^{\circ} \mathrm{C}$ for $24 \mathrm{~h}$. After 24 $\mathrm{h}$ of incubation, the zone of inhibition was investigated.

\section{CFU Measurement}

E.coli was used for measuring colony forming units (CFU) on solid medium plate. Samples treated with different concentrations of ANPs and mANPs were spread on nutrient agar plates. These samples were diluted to $10^{9}$ folds to get better colonies. After incubation at $37^{\circ} \mathrm{C}$ for $24 \mathrm{~h}$, the numbers of CFU were counted.

\section{Statistical evaluation}

Results were expressed as mean value \pm standard error of the mean of growth inhibition zones diameters. $P$ values lower than 0.05 were considered significant.

\section{RESULTS AND DISCUSSION}

The past decades witnessed the emergence of nanobiocatalysis as an increasingly attractive domain for developing novel pharmaceutical products in both science and industry, with regard to the high specificity and selectivity, and advantageous operation conditions compared to chemical catalysis ${ }^{9}$. It should be noted that quaternary ammonium compounds served as excellent disinfectants while preparing such products ${ }^{10-13}$ because their antibacterial properties involves disruption of cytoplasmic and outer membrane lipid bilayers through interaction between positively charged long quaternary nitrogen functional groups and the polar head groups of acidic phospholipids which resulted in generalized and progressive leakage of cytoplasmic materials ${ }^{7,14}$.

Agarose possesses alternating 1,3-linked $\beta$-D-galactose and 1,4-linked 3,6-anhydro- $\alpha$-L-galactose and is used extensively in gel electrophoresis experiment. Hence, in this work, efforts were raised to synthesize and characterize agarose nanoparticles followed by their stabilization by polyvinyl alcohol which resulted in the formation of uniform and spherical shaped ANPs (Fig. 1). Nanoprecipitation occurred by a rapid desolvation of agarose upon addition of dimethyl sulfoxide. Formation of ANPs is governed by Marangoni effect which involves a mass

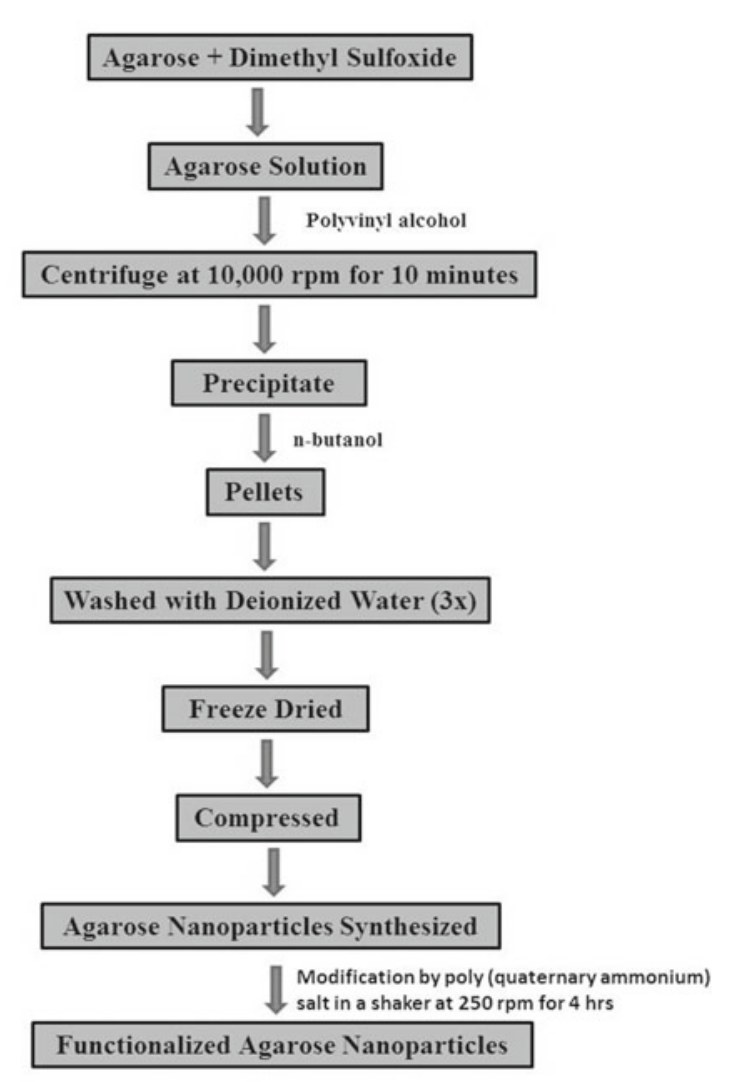

Figure 1. Flowchart representing the synthesis of ANPs. Schematic representation of agarose nanoparticles synthesized by nanoprecipitation technology.

transfer along an interface between two fluids due to surface tension gradient and interfacial turbulences ${ }^{15-17}$. The synthesized nanoparticles were characterized by XRD, TGA/DTA and TEM (Results are shown in manuscript no. BJCE-2015-0319R1 entitled "Functionalization of agarose nanoparticles by galactose for efficient production of lactose-free dairy products" accepted in Brazilian Journal of Chemical Engineering) while dynamic light scattering (Fig. 2) was used to study the size distribution and colloidal stability as an analytical tool for the characterization of ANPs. The average mean size of ANPs was observed as $32 \mathrm{~nm}$ which is under the agreement of TEM results.

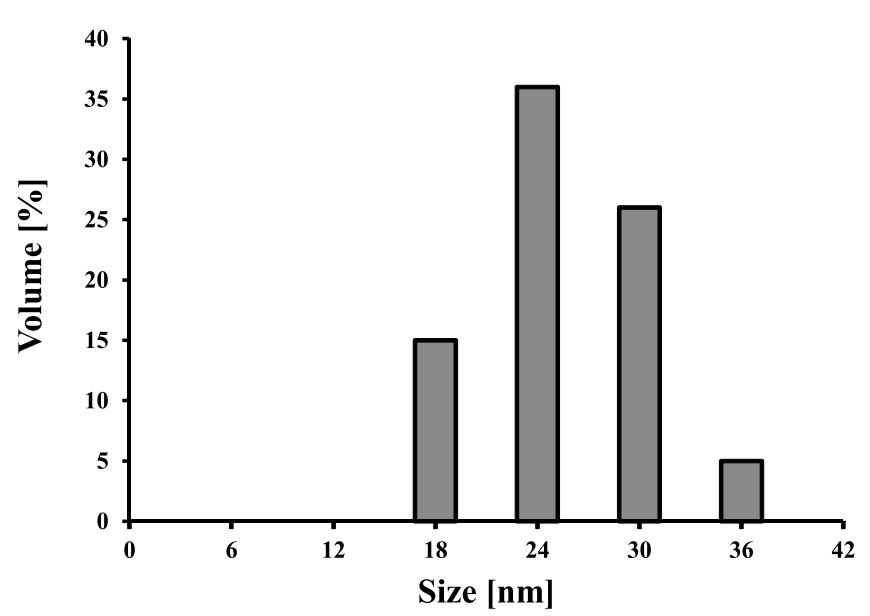

Figure 2. Differential light scattering of ANPs.

ANPs were characterized by Dynamic Light Scattering in a Malvern Zetasizer Nano ZS to observe their mean average size. 
Atomic force microscopy is a powerful tool used extensively for observing high-resolution imaging and probe interaction forces ${ }^{18-20}$. AFM image exhibited excellent nanosupport for antibacterial potential due to their small size and large surface area (Fig. 3). These molecules influence mechanical properties like stiffness and elasticity and reduce diffusion limitations to maximize the functional surface area needed for exhibiting antibacterial activity uniformly.

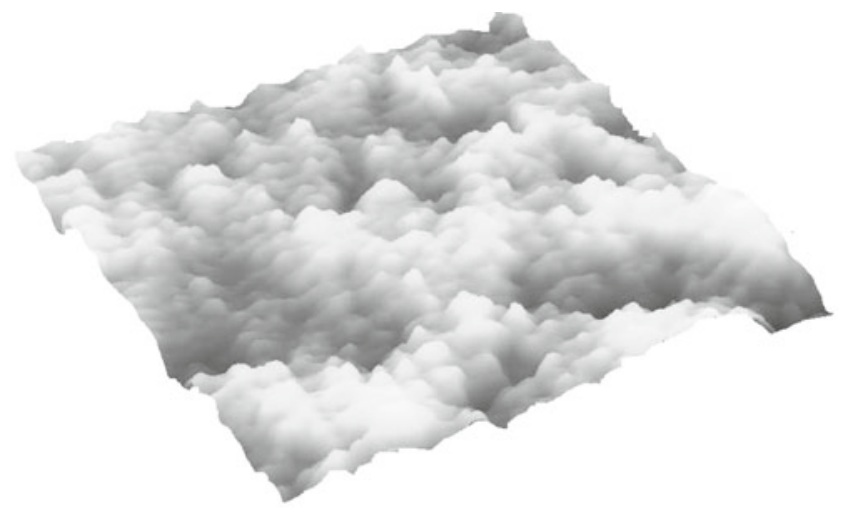

Figure 3. Atomic force micrographs of modified ANPs. Tapping mode AFM experiments of modified ANPs was performed by using commercial etched silicon tips as AFM probes with typical resonance frequency of ca. $300 \mathrm{~Hz}$ (RTESP, Veeco, Japan).

E. coli cells are ubiquitous microorganisms in the environment with high possibility of interactions with nanoparticles and finds extensive application in toxicological studies ${ }^{18}$. Moreover, S. aureus has emerged as a leading cause of colonization and infection in various soft-tissue wounds including venous leg ulcers, pressure ulcers and diabetic foot ulcers which leads to the development of chronic and non-healing wounds ${ }^{21}$. The antimicrobial activity of agarose nanoparticles was tested on gram negative bacteria, E. coli and gram positive bacteria, $S$. aureus. Antimicrobial activity of agarose nanoparticles is attributed to the increased chemical reactivity obtained due to their higher surface area to volume ratio. Figure 4

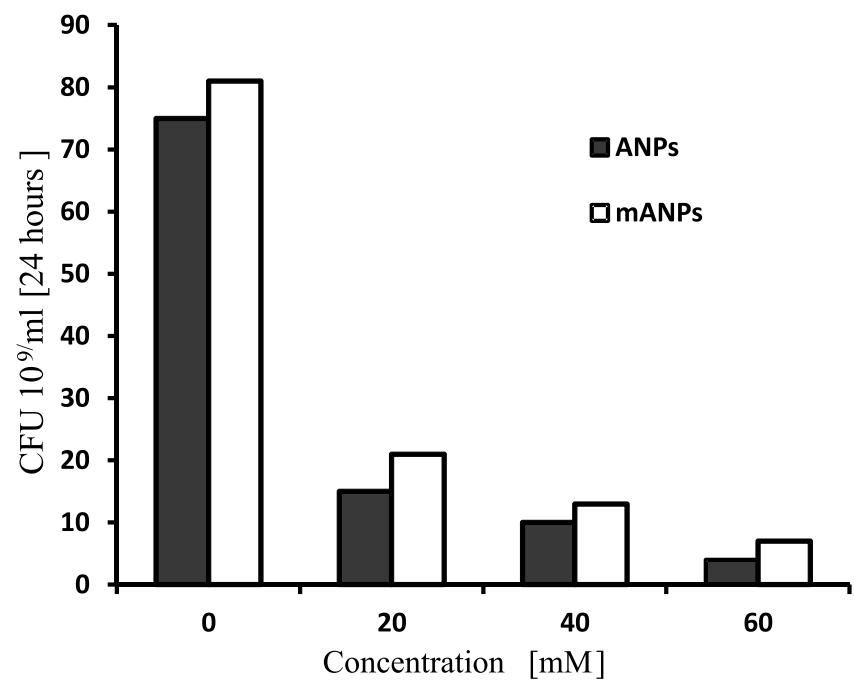

Figure 4. Antimicrobial characterization by $\mathrm{CFU}$ as a function of ANPs concentration on agar plates.

Bacterial colonies were grown on nutrient agar plates as a function of concentration of ANPs. The numbers of CFU have been observed to reduce significantly with the increasing ANPs loading.

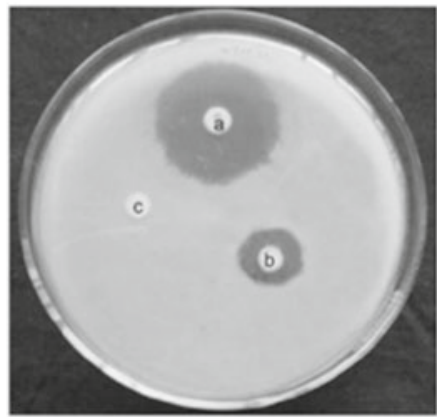

E coli

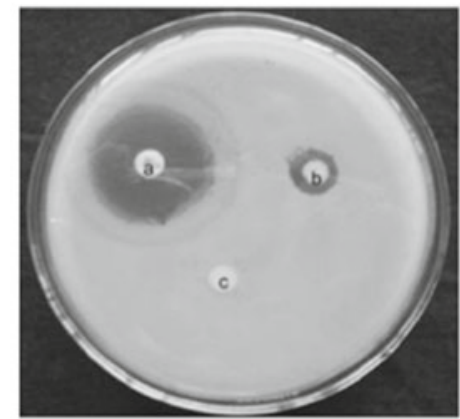

S aureus

(a)

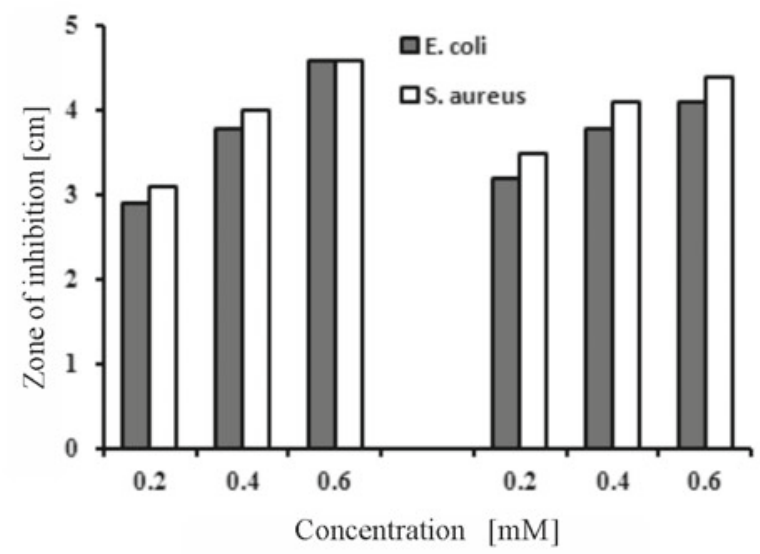

(b)

Figure 5. Zone of inhibition of antibacterial test of ANPs. Zone diameter was measured using an antibiotic zone measuring scale (HIMEDIA).

showed the plot of number of bacterial colonies grown on nutrient agar plates as a function of concentration of agarose nanoparticles. It was observed that ANPs showed $15 \times 10^{9} / \mathrm{ml}$ CFU after 24 hours of incubation at $20 \mathrm{mM}$ ANPs concentration while the modified ANPs exhibited $21 \times 10^{9} / \mathrm{ml}$ CFU under similar incubation conditions. Moreover, zone of inhibition (ZOI) was 2.9 and $3.8 \mathrm{~cm}$, respectively for $E$. coli by ANPs at 0.2 and $0.4 \mathrm{mM}$, respectively while it was 3.2 and $3.8 \mathrm{~cm}$ respectively by modified ANPs under similar conditions. Similarly, ZOI for $S$. aureus by ANPs at 0.2 and $0.4 \mathrm{mM}$ was observed at 3.1 and $4.0 \mathrm{~cm}$, respectively, while these values were 3.5 and $4.1 \mathrm{~cm}$, respectively for modified ANPs under similar incubation conditions (Fig. 5). The antibacterial activity of both ANPs and modified ANPs were increased with the increase in their concentration. Needless 
to mention, the activity was greater for modified ANPs as compared to naïve ANPs.

\section{CONCLUSION}

Significant progress was made to safely exploit the antimicrobial property of agarose nanoparticles without jeopardizing human health, critical infrastructure and environment. Additionally, great biocompatibility of ANPs makes them suitable candidate for protein and peptide drug delivery. These findings provide fundamental information for interpreting the biological interactions of nanoparticles for diverse applications in biology and chemistry. Hence, these nanoparticles can find application in the development of new pharmaceutical products as well as in self-cleaning surfaces, waste water treatment, Lab-on-a-Chip devices and many more.

\section{ACKNOWLEDGEMENT}

The authors are thankful to Prof. Waseem Ahmad for providing valuable suggestion in preparing the manuscript.

\section{LITERATURE CITED}

1. Ansari, S.A. \& Husain, Q. (2012). Potential applications of enzymes immobilized on/in nano materials: A review. Biotechnol. Adv. 30(3), 512-523. DOI: 10.1016/j.biotechadv.2011.09.005. 2. Chibber, S., Ansari, S.A. \& Satar, R. (2013). New vision to $\mathrm{CuO}, \mathrm{ZnO}$, and $\mathrm{TiO}_{2}$ nanoparticles: their outcome and effects. J. Nan. Res. 15(4), 1-13. DOI: 10.1007/s11051-013-1492-x.

3. Rao, J.P. \& Geckeler, K.E. (2011). Polymer nanoparticles: Preparation techniques and size-control parameters. Prog. Polym. Sci. 36(7), 887-913. DOI: 10.1016/j.progpolymsci.2011.01.001.

4. Nitta, S.K. \& Numata, K. (2013). Biopolymer-based nanoparticles for drug/gene delivery and tissue engineering. Int. J. Mol. Sci. 14(1), 1629-1654. DOI: 10.3390/ijms14011629. 5. Nair, L.S. \& Laurencin C.T. (2007). Biodegradable polymers as biomaterials. Prog. Polym. Sci. 32(8-9), 762-798. DOI: 10.1016/j.progpolymsci.2007.05.017.

6. Zhang, H., Wang, D., Butler, R., Campbell, N.L., Long, J., Tan, B., Duncalf, D.J., Foster, A.J., Hopkinson, A., Taylor, D., Angus, D., Cooper, AI. \& Rannard, S.P. (2008). Formation and enhanced biocidal activity of water dispersable organic nanoparticles. Nat. Nanotechnol. 3(3), 506-511. DOI: 10.1038/ nnano.2008.188.

7. Wan, W. \& Yeow, J.T.W. (2012). Antibacterial properties of poly (quaternary ammonium) modified gold and titanium dioxide nanoparticles. J. Nan. Nanotechnol. 12(6), 4601-4606. DOI: http://dx.doi.org/10.1166/jnn.2012.6147.

8. Blackburn, C.D. \& Davies, A.R. (1994). Development of antibiotic-resistant strains for the enumeration of foodborne pathogenic bacteria in stored foods. Int. J. Food Microbiol. 24(1-2), 125-136. DOI: 10.1016/0168-1605(94)90112-0.

9. Rizzello, L., Cingolani, R. \& Pompa, P.P. (2013). Nanotechnology tools for antibacterial materials. Nanomed. 8(5), 807-821. DOI: $10.2217 / \mathrm{nnm} .13 .63$.

10. Denyer, S.P. \& Stewart, G.S.A.B. (1998). Mechanisms of action of disinfectants. Int. Biodet. Biodegrad. 41(3-4), 261-268. DOI: 10.1016/S0964-8305(98)00023-7.

11. Chen, C.Z., Beck-Tan, N.C. \& Cooper, S.L. (1999). Incorporation of dimethyl-dodecyl ammonium chloride functionalities onto poly(propylene imine) dendrimers significantly enhances their antibacterial properties. Chem. Commun. 16(5), 1585-1586. DOI: 10.1016/S02684-8305(99)00048-7.
12. Chen, C.Z., Beck-Tan, N.C., Dhurjati, P.T.K., van Dyk, R.A., Larossa, P. \& Cooper, S.L. (2000). Quaternary ammonium functionalized poly(propylene imine) dendrimers as effective antimicrobials: structure-activity studies. Biomacromol. 1(3), 473-480. DOI: 10.1021/bm0055495.

13. Ioannou, C.J., Hanlon, G.W. \& Denyer, S.P. (2007). Action of disinfectant quaternary ammonium compounds against Staphylococcus. Antimicrob. Ag. Chemother. 51(1), 296-306. DOI: 10.1128/AAC.00375-06.

14. McBain, A.J., Ledder, R.G., Moore, L.E., Catrenich C. \& Gilbert, P. (2004). Effects of quaternary-ammonium-based formulations on bacterial community dynamics and antimicrobial susceptibility. Appl. Environ. Microbiol. 70(6), 3449-3456. DOI: 10.1128/AEM.70.6.3449-3456.2004.

15. Wang, N., Wu, X.S. \& Mesiha, M. (1995). A new method for preparation of protein-loaded agarose nanoparticles. Pharmacol. Res. 12(3), 257. DOI: 10.1016/j.nano.2005.12.003. 16. Wang, N. \& Wu, X.S. (1997). Preparation and characterization of agarose hydrogel nanoparticles for protein and peptide drug delivery. Pharm. Dev. Technol. 2(2), 135-142. DOI: $10.3109 / 10837459709022618$.

17. Kunkel, J. \& Asuri, P. (2014). Function, structure and stability of enzymes confined in agarose gels. Plos One 9(5), e86785. DOI: 10.1371/journal.pone.0086785.

18. Zhang, X., Yan, S., Tyagi, R.D. \& Surampalli, R.Y. (2011). Synthesis of nanoparticles by microorganisms and their application in enhancing microbiological reaction rates. Chemosphere 82(4), 489-494. DOI: 10.1016/j.chemosphere.2010.10.023.

19. Doktycz, M.J., Sullivan, C.J., Hoyt, P.R., Pelletier, D.A., Wud, S. \& Allison, D.P. (2003). AFM imaging of bacteria in liquid media immobilized on gelatin coated mica surfaces. Ultramicroscopy 97(1-4), 209-216. DOI: 10.1016/S03043991(03)00045-7.

20. Brayner, R., Ferrari-Iliou, R., Brivois, N., Djediat, S., Benedetti, M.F. \& Fievet, F. (2006). Toxicological impact studies based on Escherichia coli bacteria in ultrafine $\mathrm{ZnO}$ nanoparticles colloidal medium. Nano Lett. 6(4), 866-870. DOI: $10.1021 /$ nl052326h.

21. Kim, M.H., Yamayoshi, I., Mathew, S., Liln, H., Nayfach, J. \& Simon, S.I. (2013). Magnetic nanoparticle targeted hyperthermia of cutaneous Staphylococcus aureus infection. Ann. Biomed. Eng. 41(3), 598-609. DOI: 10.1007/s10439-012-0698-x. 